\title{
Th4-2
}

\section{Distributed optical fibre sensing in synthetic fibre ropes and cables}

\author{
D Uttamchandani, B Culshaw \\ Dept of Electronic and Electrical Engineering, University of Strathclyde, \\ 204 George Street, Glasgow G1 1XW, UK, Tel: + 441415482211 Fax: + 441415531955 \\ M S Overington, M Parsey \\ Tension Technology International Ltd, Arbroath Business Centre, \\ Dens Road, Arbroath DD11 1RS, UK, Tel: + 441241430084 , Fax: + 441241431090 \\ M Facchini, L Thévenaz \\ EPFL Swiss Federal Institute of Technology - Metrology Laboratory \\ CH-1015 Lausanne, Switzerland, Tel: +4121 6934774 Fax: +41 216932614
}

\begin{abstract}
Distributed strain measurements on synthetic fibre ropes as used in marine applications are reported. The ropes incorporate single mode fibre-optic sensors for strain measurement. A Brillouin-amplification-based distributed strain measuring system has been utilised to interrogate the fibre sensors incorporated into a parallel yarn aramid rope. Initial results are presented to conclusively demonstrate the technical feasibility of the approach
\end{abstract}

\section{INTRODUCTION}

Load bearing structures such as ropes and cables used in marine, civil and structural engineering applications are increasingly being manufactured from very fine fibres made from synthetic materials. During use, such ropes are subject to high strains which can lead to internal damage, for example through breakage of individual fibres. This may compromise the designed load bearing capacity of the rope, i.e. a reduction in effective local modulus (stiffness). Detection of such damage is important, but has proven to be difficult and unreliable, even during laboratory testing. We have initiated a project concerned with developing a "smart" rope with in-built sensors for measuring the strain distribution in the rope. Abnormal strain readings from the sensors would be indicative of possible damage to the rope, compromising its load bearing capacity. The key requirement of a "smart" rope is the ability to measure the strain distribution along its entire working length. This requirement can be met by the integration of a distributed fibre optic sensor within the structure.

The aim of the work reported here has been to determine the technical feasibility of using a fully distributed optical fibre sensor for measuring strain along synthetic fibre ropes, with a view to detecting local modulus changes. The approach investigated has involved the 
incorporation of optical fibre strain sensors into samples of rope, which were subsequently subjected to tensile loading. The fibre sensors were interrogated using a Brillouin amplification based distributed strain measuring system [1], [2]. Preliminary results obtained from the experimental work are reported.

\section{BRILLOUIN SYSTEM}

Figure 1 shows the schematic of the Brillouin system. A strong light pulse, the pump, is launched into the fibre. It crosses a weak $\mathrm{CW}$ probe signal that propagates in the opposite direction. Stimulated Brillouin scattering occurs when the two signals overlap resulting in the amplification of the probe signal. The electro-optic modulator (EOM) is the key element in the setup since it is used, on the one hand, for pulsing the $\mathrm{CW}$ light from a single frequency laser to form the pump pulse, and on the other hand for the generation and frequency tuning of the probe signal. The frequency shift on the probe laser light is achieved by applying a microwave signal to the electro-optic modulator. This creates sidebands in the laser spectrum of the probe signal. When the microwave frequency is close to the Brillouin frequency shift, one of the sidebands of the probe light lies under the Brillouin gain spectrum and is amplified [2]. The Brillouin gain spectrum, modified by the fibre strain, is determined by simply sweeping the microwave frequency applied to the modulator and recording the probe intensity.

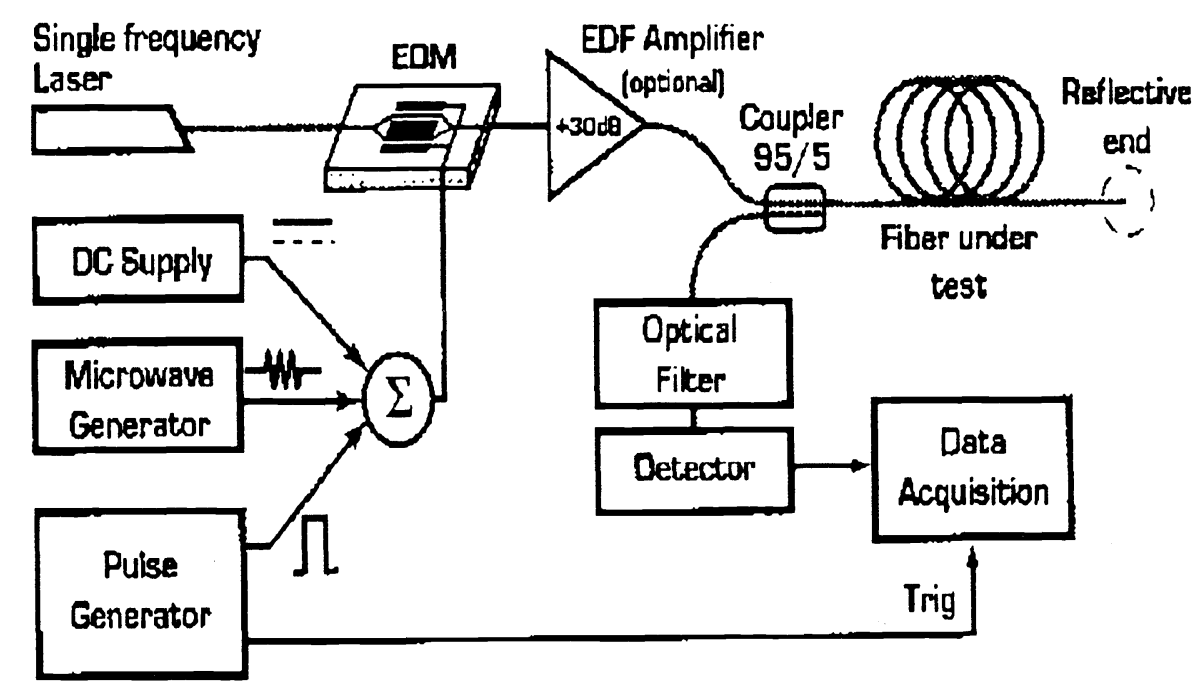

Figure 1. Schematic of Brillouin strain measuring system

\section{RESULTS FROM BRILLOUIN SYSTEM}


A length of parallel yarn aramid (Kevlar) rope with a continuous length of optical fibre embedded at the core (laid parallel to the yarns, i.e. at $0^{\circ}$ lay) was used for the experimental work. The optical fibre was not bonded to the aramid filaments. The rope had a calculated break strength (CBS) of $60 \mathrm{kN}$. The rope was subject to load increments, and at each step the fibre optic sensor was interrogated by the Brillouin system. The extension of the rope was measured separately. Figure 2 presents the post-processed data from a series of straining tests. The test machine provided data on the calibrated rope extension and could produce loads to 40 tonnes. The first half of the trace of Figure 2 shows results from a length of reference fibre which always remained unstrained. Loading was applied to the rope incorporating the fibre sensor which, in Figure 2, begins after the 21 metres point.

The load tests followed the following sequence - the corresponding results are also described:

- The initial bias trace (annotated as $3 \mathrm{kN}$ ) shows that the optical fibre incorporated in the rope was initially in compression.

- The second trace (annotated as $10 \mathrm{kN}$ ) shows that the unbonded optical fibre was able to pick up virtually all the rope strain - an extension of $58.8 \mathrm{~mm}$ on a reference length of $15.3 \mathrm{~m}$ is equivalent to $0.38 \%$ strain and the Brillouin system reported an increase from $-0.13 \%$ to $+0.22 \%$.

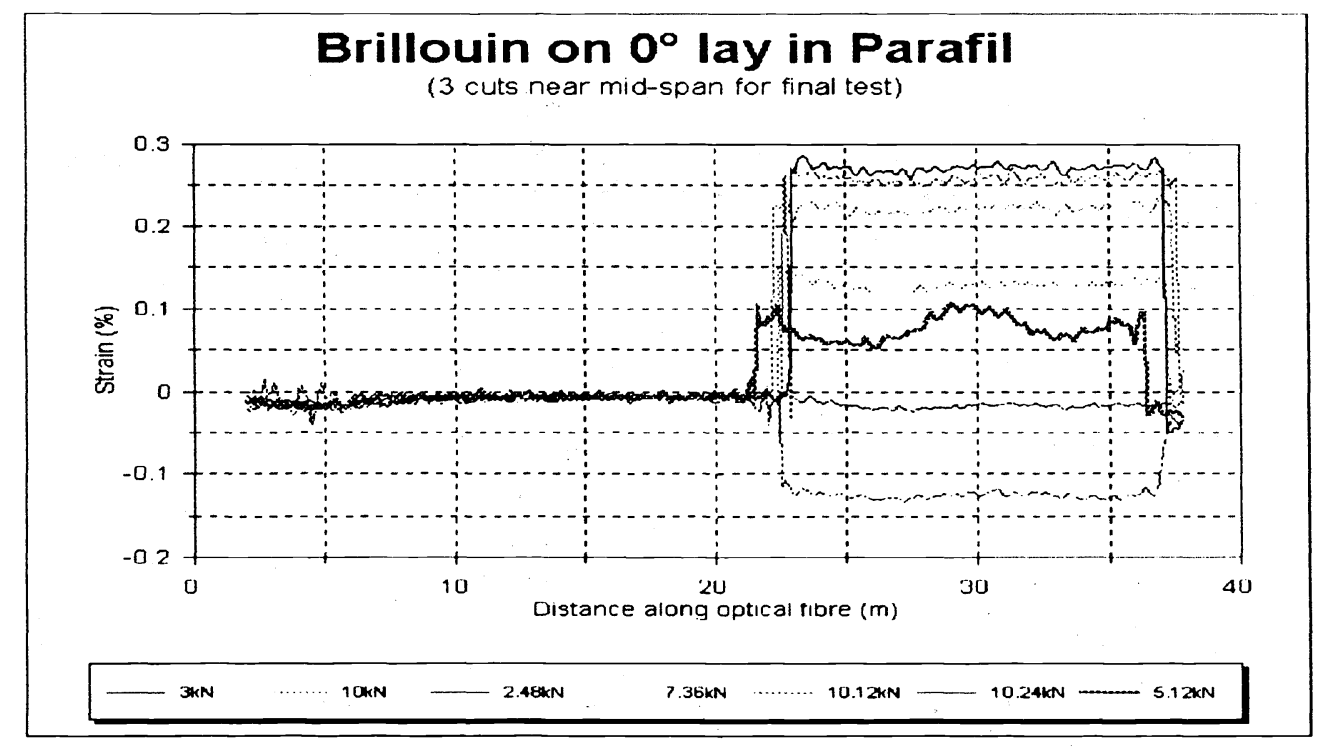

Figure 2. Monitoring of strain on small parallel yarn aramid sample

- The third trace (annotated as $2.48 \mathrm{kN}$ ) clearly shows that simply applying and removing the load once has removed most of the initial optical fibre compression. This behaviour is a typical synthetic rope bedding in process. 
- The fourth trace (annotated as $7.36 \mathrm{kN}$ ) again shows that the unbonded optical fibre was able to pick up virtually all the rope strain - an extension of $24 \mathrm{~mm}$ on a reference length of $15.3 \mathrm{~m}$ is equivalent to $0.16 \%$ strain and the Brillouin system reported an increase from $-0.02 \%$ to $+0.13 \%$.

- The fifth trace (annotated as $10.12 \mathrm{kN}$ ) still shows that the unbonded optical fibre was able to pick up virtually all the rope strain - an extension of $47 \mathrm{~mm}$ on a reference length of $15.3 \mathrm{~m}$ is equivalent to $0.31 \%$ strain and the Brillouin system reported an increase from $-0.02 \%$ to $+0.27 \%$.

- The sixth trace (annotated as $10.24 \mathrm{kN}$ ) still shows that the unbonded optical fibre was able to pick up virtually all the rope strain - an extension of $51 \mathrm{~mm}$ on a reference length of $15.3 \mathrm{~m}$ is equivalent to $0.33 \%$ strain and the Brillouin system reported an increase from $-0.02 \%$ to $+0.28 \%$.

- The final trace (annotated as $5.12 \mathrm{kN}$ ) shows beyond any doubt that the Brillouin system can detect localised loss of strength (localised increase in strain) after the rope was deliberately damaged at its centre. This last result demonstrated the capabilities of the Brillouin system, in that it was able to clearly identify that the central portion of the rope was significantly weaker than the remainder.

\section{CONCLUSION}

A Brillouin amplification distributed strain sensing system has been shown to successfully measure strain in specially constructed rope incorporating fibre optic sensors. Several significant issues have still to be addressed before the system can be developed to its next phase. The principal ones include matching the maximum strain a standard optical fibre can accommodate (accepted to be $1 \%$ ) to the maximum strain that marine ropes and cables are subject to (around 3\% for aramid and $6 \%$ for polyester) and ensuring compatibility with underwater operation, often to very high hydrostatic pressures. This requires careful design of the rope and the introduction of intermediate layers between the single mode fibre optic and the rope fibres. The anticipated applications lie in monitoring very high strength (1000 tonne and over) safety critical ropes and moorings.

\section{REFERENCES}

1. T Kurashima, T Horiguchi, H Izumita, S Furukawa and Y Koyamada, "Brillouin optical fibre time domain reflectometry", IEICE Trans. On Comms., 1993, vol E76B, pp382-390

2. L Thevenaz, M Nikles, A Fellay, M Facchini, P Robert, "Applications of distributed Brillouin fibre sensing", International Conference on Applied Optical Metrology, 1998, SPIE vol.3407, pp.374-381 